\title{
Modern and Differential Diagnosis Evaluation in Neurocognitive Disorders
}

\author{
Irene Davidescu* \\ Department of Neurosciences, University of Medicine and Pharmacy Carol Davila, Romania
}

Submission: January 28, 2019; Published: March 26, 2019

*Corresponding author: Irene Davidescu, University of Medicine and Pharmacy Carol Davila, Clinical Neurosciences, Colentina Clinical Hospital, Bucharest, Romania

Abstract

Nowadays, worldwide, around 50 million people have dementia. Every year, there are nearly 10 million new cases and as increasing life expectancy is a reality, neurocognitive spectrum is rising in incidence and it is estimated according WHO that the total number of people with dementia will reach 82 million in 2030 and 152 in 2050 worldwide, so it is a real public health problem. In 2015, the total global cost of dementia was estimated to be US\$ 818 billion and by 2018, this will rise above a US\$ trillion. In May 2017, the World Health Assembly endorsed the Global action plan on the public health response to dementia 2017-2025 [1].

It is not easy to assess the type of neurocognitive troubles, as very frequent there are overlapping signs and symptoms, psychiatric as well as neurological ones, so a holistic point of view, both as neurologist and psychiatrist will be provided.

Differential diagnosis between minor and major neurocognitive disorders as DSM 5 rules and what is new in assessing this type of pathology, with clinical cases presented for teaching purposes (Alzheimer disease, Fronto-Temporal Dementia, Dementia with Lewy Body, and other etiologies as Parkinson Disease, Multiple Sclerosis, Infectious disease -Treponema pallidum, HIV infection, etc) Clinical signs and symptoms in different stages of the disease and various etiologies, as clinical diagnosis is often crucial. Imaging tests and biomarkers, as well as neuropsychological testing will be discussed.

Treatment: symptomatic as well as social interventions will be provided.

Keywords: Neurocognitive disorders, Neurological signs, Psychiatric symptoms, Differential diagnosis, Clinical assessment

\section{Introduction}

\section{Definition}

Dementia is the term that describes the existence of a progressive cognitive decline, severe enough to interfere with daily activities, causing the socio-professional impairment of the individual and the ability to perform everyday activities. Basically, we encounter a group of symptoms, including those in the memory sphere, but also behavioral and personality disorders. Alzheimer's disease is the most common cause (60-70\% of cases), followed by vascular dementia, Lewy body dementia, Fronto-temporal dementia, and other conditions that can cause dementia patients (Parkinson's disease, multiple sclerosis in advanced stages, etc.).It must be emphasized that although dementia affects especially older people, it is not a part of normal ageing.

\section{Neurocognitive disorders in modern era \\ Epidemiology}

There are over 50 million people in the world with neurocognitive disorder. Each year, approximately 10 million new cases are diagnosed, and with the increase in life expectancy (average life expectancy worldwide in 1990 was 64.2 years and in 2015 the same indicator was 71.4 years) and the global phenomenon of population aging, the spectrum of neurocognitive disorders will increase as incidence, being estimated by WHO that the total number of people affected will reach 82 million in 2030 and 152 million in 2050 worldwide. $58 \%$ of those affected live in low-income and medium-income countries and are expected to increase to $68 \%$ by 2050 . This phenomenon generates a major public health problem, with healthcare systems in all countries facing hard pressures generated by the costs of these patients' care. For example, in 2015, global costs for people affected by dementia rose to US \$818 billion worldwide, as physical, psychological, social, and economical impact bear not only on the patients but also on the families, caregivers and in fact on all society. This amount was equivalent to $1.1 \%$ of global Gross Domestic Product (GDP) [2].

For example, in 2010, in Romania it was estimated that there were approximately 270,000 people suffering from dementia. However, of these, only 35,000 had been officially diagnosed, 
but most of the time this diagnosis occurs in late-stage disease; also, the organized social services provided for the care of these patients are in some regions insufficient and often poorly organized, with the most often returning to families, and where there is no family, patients often become so-called "social cases" striking the activity of the neurology and psychiatry, as there are no sufficient centers where they could be cared for and treated, and the costs of supervising and caring for such patients are not negligible.

\section{Diagnosis}

The assessment of neurocognitive disorders is not easy, especially since there is often overlapping of signs and symptoms, both psychiatric and neurological, that must be controlled therapeutically; patients often have other comorbidities, taking into account possible drug interactions. In 2013, with the advent of DSM-5 (Diagnostic and Statistical Manual of Mental Disorders - Fifth Edition), which reviewed the classification of mental pathology, the chapter on Delirium, dementia and other amnestic and cognitive syndromes in DSM-IV-TR 2000) is reorganized and becomes the chapter of "Neurocognitive Disorders" [3]. One of the declared purposes of the new DSM-5 in 2013 was the reduction of stigma in psychiatry. With regard to the dementia chapter, it was considered that this term was a brutal one, burdening both patients and their families, and it was decided to replace it with the term neurocognitive disorder, less stigmatizing. Another notable change occurred with the reclassification of mental illness was the one related to the stages of the disorder: if in DSM-IV-TR we encounter mild, moderate and severe dementia, at present the classification includes subtypes of minor and major neurocognitive disorders, in which the main differentiating factor is the degree of impairment of the patient's autonomy from a social point of view; In minor cognitive impairment the patient still manages to maintain his / her independence (payment of bills, administration of medication, etc.) but with additional effort and using different compensatory strategies. For both types of neurocognitive disorder, the presence or absence of associated behavioral disorders (psychotic symptoms, agitation, apathy or the like) should be specified, which are the ones that raise the greatest problem in these patients. For the major disorder, the current severity mild (difficulty in day-to-day or financial daily activities), moderate (impairment of diet or dressing) or severe (fully dependent) activity will be specified. 5 are:

The subtypes of neurocognitive disorders listed in DSM-

a. Alzheimer's, with two clinical forms have been identified: genetically-transmitted and rarer, and spontaneous, which is the most frequent one
b. vascular,
c. fronto-temporal,
d. post traumatic,
e. Lewy body disease,

f. Parkinson's disease,

g. HIV infection,

h. substance abuse disorder,

i. Huntington's disease,

j. prion disease: Creutzfeldt-Jacob Disease (CJD)

k. other medical conditions cognitive impairment,

l. multiple etiologies and

m. unspecified neurocognitive disorders [3].

\section{Clinical diagnosis}

Is essential provided that patients are sent for examination to neurological or psychiatric physicians. Neurocognitive testing is often laborious and requires the examiner's patience, and patients are often irritable, suspicious and anxious about their memory situation, at the onset of symptoms. Patients with neurocognitive disorders are often at the junction between two specialties: neurology and psychiatry. If they have certain neurological symptoms they are addressing to the neurologist, if behavioral or mood problems are prevalent, they will be referred to the psychiatrist. It is very important that both neurologists and psychiatrists have a holistic view of the spectrum of neurocognitive disorders in order to exclude other entities that might manifest themselves in similar ways, for a correct differential diagnosis (Figure 1).

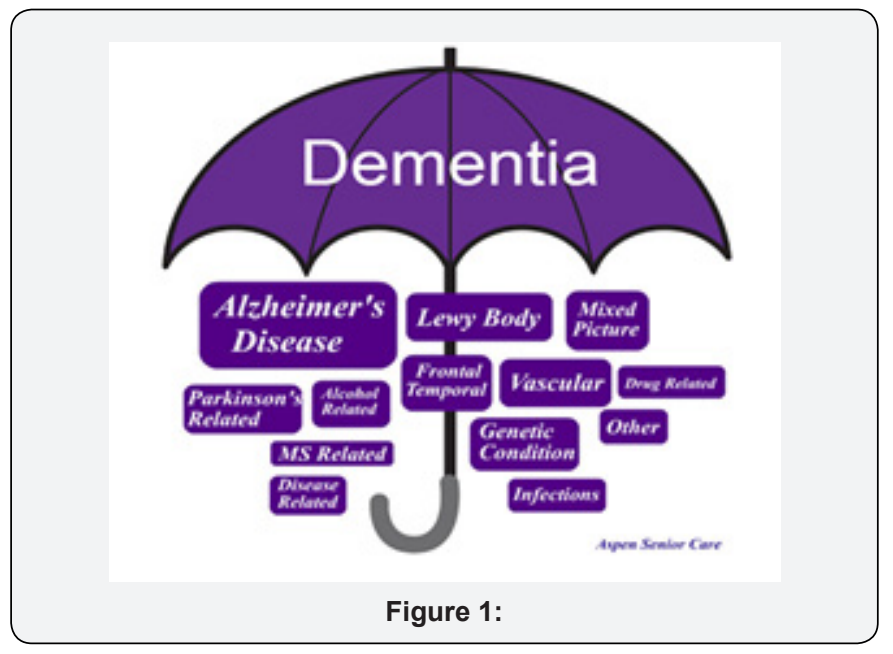

\section{Neurological symptoms and signs}

a. Memory disorders: the difficulty of remembering previously learned information and the inability to accumulate new information. The first to be lost are recent events, while old memories can be preserved.

b. Talking disorders: the patient cannot find his words anymore, even for simple notions.

c. Inability to perform different coordinated motor activities: the patient "does not know" how to properly dress, how to unlock the door with the key or other common tasks (apraxia) 
d. Failure to recognize, identify and denote common objects.

e. Disturbances in the functions of organizing daily activities, inability to make decisions.

f. Problems of abstract thinking, computational disturbances, temporal and spatial disorientation, loss of initiative.

g. False recognition: First difficulty in recognizing known physiognomies followed by erroneous identifications that can cause anxiety.

h. In some pathologies we can encounter pyramidal signs, parkinsonism, cerebellar signs, involuntary movements, etc.

i. Psychiatric symptoms and signs:

j. Agitation and physical or verbal aggression.

k. Psychotic disorders: hallucinations, usually visual, delusional ideas (of persecution, jealousy, abandonment, etc.).

l. Disorders of mood disorder: mainly depression and anxiety, rarely exaggerated euphoric states.

m. Disorders of food behavior: excessive or excessive appetite, ingestion of non-food substances.

n. Sexual disinhibition: Sexual comments, obscene gestures, rarely aggressive sex

o. Urinary and faces incontinence, performance of physiological needs in inappropriate places or in the presence of other people.

Regarding the delusional ideas, these are beliefs, false ideas, unshakable, in which the patient believes regardless of the contrary evidence that is brought to him. The most common ones are

a. the idea that people around you talk about the patient;

b. ideas of theft, the patient is convinced that things are stolen or hidden; many times, such beliefs arise as a result of memory disorders (the patient completely forgets where he put his things and accuses others of having taken them);

c. The ghost tenant - the idea that other people live in the house may also appear because of visual hallucinations of people;

d. Patient's belief that he is persecuted, that he is wicked;

e. ideas of abandonment - the patient thinks he is going to be left out of the family, driven out of his own house; there are also situations where such ideas have a real basis (the cases of elder abuse);

f. delusional ideas of jealousy - the conviction that the spouse has an extra conjugal relationship; they can also occur due to visual hallucinations (the patient sees foreign people in the house).

All neurological and psychiatric symptoms and signs are characterized by a gradual onset, the patient develops certain strategies to conceal their difficulties, for some time retains a deceptive "facade", but the decline progressively worsens. It is very important that during anamnesis, to assess all other associated diseases, evolution of symptoms, and the interview must be extended to families other people who can provide comparative data about the anterior cognitive level and the time course of symptoms, as well as every day skills [4].

\section{Diagnosis}

a. In the initial diagnosis stage, biological and imaging investigations (nuclear magnetic resonance, computed tomography) are required to detect any other organic causes that may cause neurocognitive disorder.

b. Clinical general examination may reveal signs of a general condition accompanying dementia like a metabolic disorder, hypothyroidism, severe anemia, tumors, etc.

c. Neurological examination is mandatory as various neurological signs may guide the diagnosis to primary neurological diseases which can associate dementia, like Wilson's disease, Creutzfeldt-Jacob disease, Parkinson disease, etc. Also, neurological examination is very important to distinguish in differential diagnosis, Alzheimer's dementia and vascular dementia.

d. Psychiatric assessment is very important also as it may reveal non-cognitive signs: psychiatric and behavioral symptoms which are often present from the early stages of the disease, like disinhibition and agitation, affective disorders including depression and anxiety, psychotic phenomena, confusional states, obsessive episodes.

e. Neuro-psychological examinations must encompass test as Mini Mental state Examination (MMSE), Montreal Cognitive Assessment (Mocap) for differential diagnosis of mild cognitive impairment, as well as Sunderland Clock Test. Neuropsychiatric Inventory (NPI) as well as assessment of Activity of Daily Living (ADL) are also important for rating. The cognitive subscale of the Alzheimer's Disease Assessment Scale (ADAS-Cog) is used also in selected cases, as it is not a routine test in everyday practice, beeing the most frequently used tests to measure cognition in research studies and clinical trials for new drugs and other interventions.

\section{Laboratory examinations}

usual tests, including thyroid hormones, serum vitamin B12 or homocysteine in selected cases

serological tests for infectious diseases like AIDS, syphilis, borreliosis, herpetic encephalitis, or other suspected infections immunological tests for diagnosis of vasculitis, systemic lupus, 
or other clinical entities that may develop altered cognitive state toxicological tests (for identification of heavy metal intoxications) genetic tests for identification of early familial Alzheimer's disease, front-temporal dementia, CADASIL, which have genetic mutations.

\section{Cerebrospinal fluid (CSF) examination in selected cases:}

a. In Alzheimer disease the Aß42 peptide is low and tau protein is elevated in the CSF compared to non-dementia subjects of the same age [5].

b. In case of Creutzfeldt-Jakob disease suspicion (rapid programmable dementia associated with presence of myoclonus), CSF dosing of 14-3-3 protein is important for diagnosis and has proven to be a reliable and stable biomarker for CJD beeing included in the WHO diagnostic criteria [6].

c. Neuroimaging Imaging should be used to exclude nondegenerative, nonvascular pathology such as tumors, subdural hematoma, hydrocephalus, etc., as well as cerebrovascular disease as the major pathological substrate for the cognitive problems (like vascular dementia or MCI of the vascular type) or other degenerative dementias presenting with focal or lobar atrophy. Either MRI or CT may be adequate for the first issue above; MRI is superior in assessing the other two categories [4].

d. CT examination: Use of CT imaging for early diagnosis of $\mathrm{AD}$ should be the focus of future research for those individuals who cannot receive MRI [4].

e. MRI examination: Field strength of $1.5 \mathrm{~T}$ is adequate and embraces the combined attributes of wide use and availability. Coronal brain imaging, preferably perpendicular to the long axis of the hippocampus, should be included in routine MRI protocols. Analyses should be standardized and include a measure of global loss and a measure of medial temporal lobe volume [5].

f. Positron Emission Tomography (PET) is an imaging technique that provides information about physiological and biochemical processes. 18F-fluorodeoxyglucose (FDG) is the most extensively used PET tracer in the study of Alzheimer's Disease (AD). Patients with AD have characteristic reductions in FDG PET measurements of regional brain activity, which are progressive and correlate with dementia severity. An abnormal PET scan may provide supportive evidence for the presence of a neurodegenerative dementing disease, rather than a reversible psychiatric illness or symptoms due to normal aging. PET should be considered as an option to assist in the recognition of dementia when coexisting illness or baseline abilities and cooperation are uncertain [4].

g. Single Photon Emission Computed Tomography (SPECT) is another molecular imaging technique that can provide information similar to that obtained with PET. Because of differences in the physics of single photon emissions as compared with positron emissions, SPECT has lower resolution than PET. The inherent superiority of PET probably makes this technique preferable, although SPECT can provide clinically useful information that may be comparable, as SPECT imaging has received widespread clinical application because of its simplicity, use of long-lived radio-nuclides, and lack of need for a local cyclotron [4].

h. Electroencephalography (EEG) may help to differentiate between Alzheimer Disease, subjective complains and psychiatric diagnoses. EEG is recommended in differential diagnosis of atypical clinical presentations of neurocognitive disorders and it can also provide early evidence for CJD; it can also suggest the possibility of a toxic-metabolic disorder, transient epileptic amnesia or other previously unrecognized seizure disorder [4].

\section{Differential Diagnosis}

\section{With other cerebral or general conditions}
a. Head trauma
b. Normal Pressure Hydrocephalus
c. Problems with Vision and Hearing
d. Liver and Kidney Disease
e. Hormone Disruption
f. Infections
g. Toxic metals
h. Cancer

From the practical point of view, we can classify neurocognitive disorders in some categories:
a. Permanent and progressive:
b. Alzheimer Disease
c. Vascular dementia
d. In Parkinson Disease
e. Lewy Body dementia
f. Fronto-temporal dementia
g. Huntington disease
h. Hallervorden-Spatz
i. Progressive supranuclear Paralysis
j. Multiple sclerosis
k. AIDS dementia complex
l. Neurosyphilis
m. Creutzfeldt-Jakob disease 

n. Permanent but without progressivity
o. Post traumatic dementia
p. Post anoxia dementia
q. Partially or completely reversible
r. drugs and toxics: alcohol, carbon monoxide, lead, mercury, manganese, pesticides, trihexyphenidyl, barbiturates, tricyclic antidepressants, lithium, digital, cocaine, etc.
s. infectious diseases: encephalitis, meningitis, tuberculosis, parasitosis, neuroborreliosis)
t. internal normotensive hydrocephalus
u. subdural hematoma
v. cerebral tumors
w. Wilson disease

Metabolic disease: chronic kidney failure, dialysis dementia, hepatic impairment, chronic hypoglycemia

a. Endocrine disorders: hypothyroidism, Cushing's syndrome [8]

b. Autoimmune Disorders: Systemic Lupus Erythematosus with associated cerebral vasculitis

c. Various deficiencies: Wernicke-Korsakoff syndrome, deficiency of B12 vitamin and folic acid, niacin deficiency (pellagra)

d. Paraneoplastic syndromes: limb encephalitis, other autoimmune encephalitis

\section{Treatment}

Early therapeutic intervention with existing symptomatic drugs (acetyl-cholinesterase inhibitors and NMDA receptor antagonists) may slow the increase in memory impairment; all other associated illnesses should be treated correctly, especially those with a tired impact (high blood pressure, diabetes mellitus, dyslipidemia, etc.).
a. acetyl-cholinesterase inhibitors
b. Donepezil -5-10 mg daily
c. Rivastigmine -6-12 mg daily
d. Galantamine-16-24 mg daily
e. N-methyl-D aspartate (NMDA) receptor antagonist
f. Memantine - $20 \mathrm{mg}$ daily

Treating behavioral and psychiatric symptoms may ease the patient's and families burden, as these symptoms are often the most upsetting, but medical treatment of non-cognitive symptoms is recommended only in situations where nonpharmacological approaches are not possible or not effective.
Choosing psychotropic medication, especially antipsychotic medication, should be done following an individual review, with family or caregiver consultation about the possible benefits or risks of treatment. Treatment should be limited, regularly reviewed, targeted for specific symptoms, and evaluated periodically, as short time as possible [8].

\section{Agitation}

Trazodone can be very helpful for reducing behavioral disturbances, because of its sedating qualities [9].

Mood stabilizers like sodium valproate or carbamazepine can be used alone or in combination with an antipsychotic drug $[10,11]$.

Benzodiazepines should be used only with great caution if at all because they can cause confusion, excessive day-time sedation, increased falls as well as paradoxical reactions in some cases.

\section{Psychotic symptoms}

Newer atypical antipsychotic drugs like risperidone, quetiapine, olanzapine or clozapine may be used but with caution and taking into account other associated diseases; it is preferable to use them for short term care.

\section{Depression}

Selective Serotonin Reuptake Inhibitors are of choice and therapy should be started as soon as depression is suspected.

\section{Sleep disturbances}

non-benzodiazepine sedative hypnotics as zolpidem trazodone. As cognitive impairment advances, psychosocial strategies and interventions need to be better organized to provide patients with psychological comfort and quality of life. A lot of structured cognitive programs provided by medical and social staff with appropriate training and qualifications can ease patients' burden as well as caregivers one.

\section{Future Strategy}

In May 2017, the World Health Organization Assembly adopted the Global Dementia Public Health Plan for the period 2017-2025 stating that dementia is a public health priority [12]. All countries involved have an obligation to develop and implement health strategies and policies to address these patients, ensuring equality, dignity and human rights, as well as helping all those involved in caring for these patients (families, social workers, non-governmental associations etc.). There is a need for multi-sectorial collaboration between the medical and psychosocial services for the early diagnosis, assessment, monitoring and care of these patients.

The recommendation of this Global Plan is to allocate sufficient financial resources for the implementation of national action plans in the field of dementia, health, social services, education and labor market. Patients with dementia have a 
complexity of needs and high levels of dependency but also multiple comorbidities, especially in the final stages of illness, requiring a multitude of health and social services, including long-term care / palliative care at the end. All processes of diagnosis, treatment (pharmacological and psychosocial), rehabilitation and palliative should be of public interest and the recommendation of the WHO Global Strategy Plan is the implementation of multi-sectorial care and support systems for patients affected by neurocognitive disorders as well as their caregivers, in order to increase the quality of life of both categories.

\section{The most important strategies that health systems must implement regarding dementia are [1]}

a. Policies to early diagnosis so that an optimal early management can be provided

b. Holistic management of the patients with treatment of all other accompanying physical illness

c. Correct identification and treatment of behavioral and psychological symptoms

d. Supportive measures for families and caregivers as their burden is often bigger than the patient's one

e. optimizing physical health, cognition, activity and wellbeing

\section{Conclusion}

The number of people who will be affected by neurocognitive disorders will be higher. Mental health professionals (neurologists, psychiatrists) and health systems need to be prepared to cope with the challenges of caring for a number of increasing numbers of patients in this category. The integrative approach to neurological disorders but especially psychiatric symptoms (behavioral disorders, mood disorders) should be made as targeted and earlier as possible, taking into account

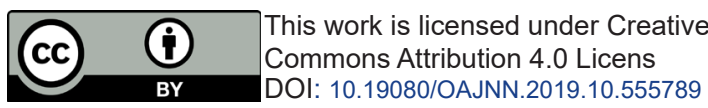

all other patients' associated illnesses, to ensure a beneficial climate for both patients and families or caregivers.

\section{Motto}

"The human brain has 100 billion neurons, each neuron connected to 10 thousand other neurons.

Sitting on your shoulders is the most complicated object in the known universe."

\section{References}

1. https://www.who.int/mental_health/neurology/dementia/action plan_2017_2025/en/

2. https://www.who.int/news-room/fact-sheets/detail/dementia

3. American Psychiatric Association: Diagnostic and Statistical Manual of Mental Disorders- (5 ${ }^{\text {th }}$ Ed) Neurocognitive Disorders: 591-643.

4. Hort J, O Brien JT, Gainotti G, Pirttila T, Popescu BO, et al. (2010) EFNS guidelines for the diagnosis and management of Alzheimer disease. Eur J Neurol 17(10): 1236-1248.

5. https://www.alz.org/national/documents/imaging_consensus_report.pdf

6. Blennow K, Hampel A (2003) Cerebrospinal fluid markers for incipient Alzheimer's disease. Lancet Neurol 2(10): 605-613.

7. Chohan G, Pennington C, Mackenzie JM, Andrews M, Everington D, Will RG, et al. (2010) The role of cerebrospinal fluid 14-3-3 and other proteins in the diagnosis of sporadic Creutzfeldt-Jakob disease in the UK: a 10-year review. J Neurol Neurosurg Psychiatry 81(11): 1243-1248.

8. (2010) Romanian Association of Neurology, Romanian Alzheimer Association: Diagnostic and Treatment Guide for Dementias; Amaltea.

9. Starkstein SE, Mizrahi R (2006) Depression in Alzheimer's disease. Expert Rev Neurother 6(6): 887-895.

10. Lonergan ET, Luxenberg J Valproate preparations for agitation in dementia. The Cochrane Database of Systematic Reviews.

11. Olin JT, Fox LS, Pawluczyk S, Taggart NA, Schneider LS (2001) A pilot randomized trial of carbamazepine for behavioural symptoms in treatment-resistant outpatients with Alzheimer's disease. Am J Geriatr Psychiatry 9(4): 400-405.

12. https://www.alz.co.uk/

Your next submission with Juniper Publishers will reach you the below assets

- Quality Editorial service

- Swift Peer Review

- Reprints availability

- E-prints Service

- Manuscript Podcast for convenient understanding

- Global attainment for your research

- Manuscript accessibility in different formats ( Pdf, E-pub, Full Text, Audio)

- Unceasing customer service

Track the below URL for one-step submission

https://juniperpublishers.com/online-submission.php 